zytenzählungen gebraucht werden können. Für diese letzteren miissen die Einteilungen sehr fein sein, wie z. B. in der ThomaZeißschen Kammer, und dafür sind so viele „Linien" erforderlich, daß man auf großen, so eingeteilten Flächen die Orientierung vollkommen verlieren würde. Das ist eben ein Nachteil, der manchen Kammern anhaftet, daß sie für Erythrozytenzählungen, die doch ebenso wichtig sind und ebenso génau ausgeführt wcrden müssen wie die Leukozytenzählungen, nur eine ganz beschränkte Fläche besitzen, sehr oft nur das Thoma-Zeißsche Quladrat.

Außerdem ist die Einteilung schon fïr Lenkozytenzählungen bci starker Vermehrung viel zll grob. Man ist dann gezwungen, zur Ermöglichung der Zählung stärkere Verdïnnungen zu machen, was immer eine große Fehlerquelle bedingt.

Das Prinzip, anf welchem bei manchen Kammern die Vergrößerung beruht - die Verlängerung der Seitenlinien des Thoma-Zeißschen Quadrates --, ist sicherlich nicht elastisch genug, um ohne Erschwerung der Orientierung noch weitere Vergrößerungen vornehmen zu können. Deswegen beschränken sich die meisten nach diesem Prinzip vergrößerten Kammern anf eine Fläche von $9 \mathrm{qmm}$, und das gilt eben als zu wenig, um genaue Zählungen zu ermöglichen. Um Anhaltspunkte für die Zählung zu bieten, können aber auch diese Kammern der doppelten Linien nicht vollkommen entbehren, und dadurch wird cbenfalls die Orientierung erschwert.

Dic Apparate, die das Zählnetz im Okular lıaben, crfüllen zwar dic Forderung der großen Fläche, dafüı aber liaben sie auch große Nachteile, vor allem den, daß sic von bestimmten Vergrößerungen und 'Tubuslängen abhängig sind, was ihre praktische Verwendbarkeit beschränkt. Sic crfordern außerdem Zusatzapparate, was sie relativ kostspielig macht.

Es scheint mir sehr vorteilhaft, unter Umstïnden anch Zählungen mit Immersion leicht vornehmen zu können, was mit groben Einteilungen nicht möglich ist - wegen des verhältnismäßig kleinen Gesichtsfeldes der Immersion - und mit feinen Einteilungen sehr schwierig ist - wegen der vielen Linien, die dazu nötig sind und die. wie erwähnt, die Orientierung sehx stören.

Iel habe daher versucltt, eine Zällkanmer zu konstruieren, die ohıe kostspielige technische Scliwierigkeiten folgende Vorteile in sich vercinigen soll:

1. Möglichst genanc und raselıe Zählungen der Lenkozyten, auch bei starker Vermehrung, und der Erythrozyten zu gestatten. Deswegen 1113 die Kanmer sehr ,gloß" sein und auch noch weiter vergrößert werden können, ohne daß die Orientierung irgendwie erschwert wird. Sie soll daher auch keine ,doppelte Linie" entlaalten.

2. Sie soll aucl mit Immersion gebraucht werden können, unı Differentialzählungen der Leukozyteı wie auch Zälılungen von kleineren Flementen (Mikroben ete.) zı ermöglichen.

Diese Bedingungen loffe ich durclı eine einfache Abänderung des Prinzips, das vielen Kammern zugrundeliegt, erreicht zu haben, indem ich die Einteilung statt auf einem Quadrat auf einen langen Rechteek volnehme.

Außerdem habe iclı die Kammer auch weniger tief machen lassen.

Aus der Rinführung eines langen Rechtecks als Einteilungsflächı

Aus der Dermatologischen Klinik der Universität in Bern (Direktor: Prof. Dr. Jadassohn).

\section{Eine Zählkammer für zytologische und bakteriologische Zwecke.}

Von Dr. Emil Liebreich, I. Assistent der Klinik.

Es liegt nicht in meiner Absicht, die jetzt in Gebranch befindlichen Zählkammern zu kritisieren; sie haben alle, die älteren wie die neueren, mit ihren vielen Abänderungen den einen gemeinschaftlichen Zweck, eine genaue und rasche Zählung zu gestatten.

Um die Zählung möglichst unabhängig von den zufälligen Unregelmäßigkeiten der Verteilung der Zellen zu machen, muß die Kammer eine große Fläche haben.

Nun stoßen alle Versuche, eine ,große Kammer" herzıstellen, auf die Schwierigkeit, daß, je größer die Kammer ist, um so mehr ,Linien" nötig werden, wodurch die Orientierung erschwert wird. Der Linien werden dann ,zu viel“", die Zählıng wird ermüdend und kann nicht mehr rasch ausgeführt werden.

Das ist auch der Grund, weshalb manche sogenannte ..großo K丸mmern" nur fïr Laukozyten- ind nicht anch fïr Erythroergcben sich zwei wichtige Vorteile:

1. Die Figur kann so viele Male wie gewünsclit wiederholt werden, sodaß man der Kammer eine beliebige Größe geben kann, olıne dædurcl die Orientierıng zu erscliweren. Um jedes Rechiteck von dem nächstoberen und näclistunteren leicht zu unterscheiden, genügt es nämlich, jedes zweite derselben durelı eine Anzalıl lorizontaler Linien zu durchqueren. Daraus resultiert Folgendes: jedes Rechteck unterscheidet sich stets von den beiden, zwisclien welchen es liegt (s. Fig. 1). Aus dieser Horizontalteilung ergibt sich noch ein weiterer Vorteil, auf den iclı unten zil sprechen komme.

2. Die Zählung kann in ciner einzigen Ricbtung: horizontal, vorgrenommen werden, ungefähr so, wie man es mit einem gewöhnlichıcn Ausstrichtrockenpräparat zu machen pflegt. Man kann sich leicht überzeugen, daß man auf diese Weise schneller zählt und weniger ermüdet.

Bei dieser Einteilung ergeben sich auch aus der geringeren Tiefe der Kammer $\left(0,025 \mathrm{~mm}=1 / \mathbf{4 0}_{0} \mathrm{~mm}\right)$ noch zwei andere Vorteile:

1. Sie gestattet anclı Zählungen mit Immersion;

2. jede Flächeneinheit nıeiner Kammer enthält, infolge ihrer geringen Tiefe, viermal weniger zu zählende Elemente als die gleiche Flücheneinheit der Kanmern von 0,1 mm Tiefe. Die nene Kammer kann also, obwoh größer, fül' den gleichen Zéllgehalt eine weitere Einteilung und damit auch die dazu nötigen linien entbehren. Ist aber eine noch feinere Einteilung nötig, wie z. B. für die Zählungen von ganz kleinen Elementen (Mikroben) and moines Erachtens mit Vorteil auch von Erythrozyten, dann kann 
Inan sie hier leicht vornehmen. Dem kleinsten abgegrenzten Raum z. B. der Thoma-ZeiBschen Kammer entspricht in meiner Kammer ein Raum mit viermal so großer Basis. Diese kann also noch weiter eingeteilt werden (in vier z. B.), ohne daB dic Linien in der feinsten Einteilung näher zusammenliegen als bei den feinsten linteilungen bei der ThomaZeißschen Kammer, wo sic ja einen Raum von viermal größerem Volumen begrenzen.

Diese feinere Einteilung ist aber hier sehon erreicht durch die erwähnten Querlinien, die zur Unterscheidung der langen Rechtecke gebraucht worden sind. Führt man deren drei, gleich weit voneinander a bstehende, für jedes zu durchquerende Rechteck ein, dann werden diese letzteren in vier gleiche Teile geteilt (s. Fig. 1 - Reihen B).

Die Höhe jedes Rechtecks beträgt $0,1 \mathrm{~mm}$ und die Länge $1 \mathrm{~cm}$; die Oberfläche also $1 \mathrm{qmm}$.

Führt man nun durch alle Rechtecke Vertikale, die $0,1 \mathrm{~mm}$ voneinander abstehen, so zerfällt jedes lange Rechteck in 100 Quadrate von $0,1 \mathrm{~mm}$ Seitenlänge (s. Fig. 1). Es entstehen also zweierlei Quadrate, dic einen sind nicht mehr eingeteilt und bilden nebeneinander lange,

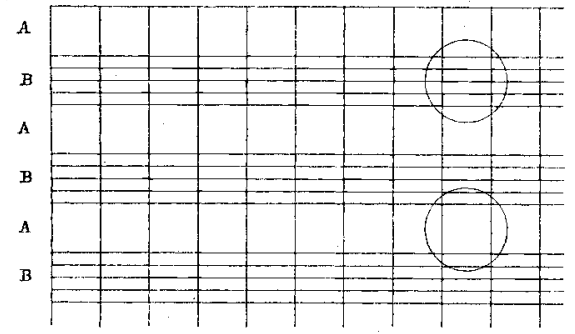

Fig.t.

Der zehnte Teil der Kanmer. Der obere Kreis. stellt ein ,,durcliquertes" Quadrat bei Okular $3-$ Trockensystem 7 dar. Der untere Kreis ein nicht durchquertes bei der gleichen Vergrößerung. nicht ,durchquerte "6 Rechtecke (Fig. 1 - A); die anderen sind noch in vier Teile geteilt und bilden lange ,durch-

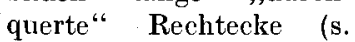
Fig. $1-\mathrm{B}$ ).

In meiner. Kanımer wird also $1 \mathrm{qmm}$ in 100 Quadrate geteilt, bei der Thoma-ZeiBschen Kammer die gleiche Fläche in 400. Dabei ist aber der Kubikinhalt der kleinen Quadrate der gleiche und damit auch die Zahl von Zellen. Die Oberfläche der feinsten Einteilung eines, ,durchquerten“ Quadrats $(=1 / 4$ eines undurchquerten Quadrats und 1/400 eines langen Rechtecks) ist also gleich der feinsten Einteilung des Thoma-Zeißsclien Quadrats, enthält aber viernal weniger Elemente als letzteres, was sich für Zählungen von Mikroben und auch von Erythrozyten als sehr praktisch elwiesen hat.

Aus dieser Einteilung ergibt sich noch ein Vorteil, der die Orientierung selir erleichtert: bei bestimmten starken Vergrößerungen (Ok. $\mathbf{3}-\mathbf{5}$; 'Trockensystem 7) und insbesondere bei Anwendung der Immersion

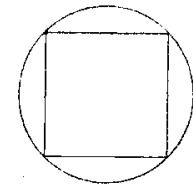

Fig.2.

Ein nicht-, durchbei Immerslon $1 /$ — Okular 3 .

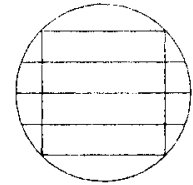

Fig.t.

Ein durchquertes Quadrat bei Inlmersion $(1 / 12)$ nimmt ein Quadrat genau oder fast genau das ganze Gesichtsfeld ein, soda $B$ man keine anderen Linien zu sehen bekommt als diejenigen, die die zu zählende Fläche umschreiben (s. Fig. 2, 3).

Wie schon erwälnt, kann dic Kammer oline Erschwerung der Orientierung leicht vergrößer't werden, indem man entweder die Rechtecke in größerer Zalıl wiederholt, oder sie verlängert, oder beides vornimmt. - Die jetzt von der Firma E. Leitz-Wetzlar liergestellte Kammer besteht aus 60 Rechtecken, jedes von 1 qmm Oberfläche. 30 dieser Rechtecke sind noch horizontal geteilt, 30 nicht. Insgesamt umfaßt also die Kammer eine Oberfläche von 60 qmm mit einem Inhalt von $1 \frac{1}{s} \mathrm{cmm}$. Die Kammer besitzt zwei Lateralrinnen, um den eventuellen ,Druckfelıler"zu vermeiden. Sie füllt sich entweder wie die Bürkersche Kammer" durch Kapillarität oder wie die anderen Kammern. Zum ersteren Zweck bringt nian das Deckglas auf den Ring del Kammer, sodaß beinahe die garıe Zählfläche außer einem kleinen Raume bedeckt wird. Mit zwei Fingern fixiert nan zwei entgegengesetzte Ränder des Deckglases und läßßt die Flüssigkeit in den frei gebliebenen Raum zufließen. Besondere Sorgfalt bei der Verteilung der Flüssigkeit ist überflüssig, da, bei der großen Fläche der Kanimer, eventuelle Fehler sich von selbst kompensierell.

Durch die geringe Tiefe der Kammer wird auch das Absetzen der Wlemente beschleunigt, was sich besonders bei Mikrobenzählungen als selir vorteilhaft (zeitersparend) erwiesen hat.

Als Pipetten und Verdünnungsflïssigkeiten dienen die üblichen.

Unı Zällungen vorzunchịen, verfährt man folgendernll a Be 11:

a) Leukozytenzählung. Sie ist möglich auch bei Objektiv 3 , Okular 3, besonders wenn man sich eines Kreuztisches bedient. Es ge11ügt dafür eine einzige Reihe (ein Rechteck). z. B. diejenige, die in der Witte des Gesichtsfeldes liegt, von einem Fnde bis zunı anderen zu ver- folgen. Bei Trockensystem 7, Okular 3, stellt man sich ebenfalls ein Ende eines Rechtecks ein. Eventuell kann man noch durch Ausziehen des Tubus (wenn man nicht genügende Vergrößerungen besitzt) die Figur so einstellen, daB ein Quadrat ganz oder fast ganz vom Kreise des Gesichtsfeldes umschrieben ist (s. Fig. 1 - Okular 3, Objektiv 6).

Man hat dann nur das Präparat horizontal zu verschieben, bis mail ans andere Ende des Rechtecks gelangt. Das erfolgt sehr rasch. Bei einem normalen Menschen, mit 7000-8000 Leukozyten pro $\mathrm{cmm}$, findet man auf einer ganzen Reihe (d. $\mathrm{l}_{\mathrm{l}}$. in einem Rechteck) 16-18 Zellen, d. h. 84-82 Quadrate sind ganz leer. Damit hat man also die Leukozy ten zall auf $1 \mathrm{qmm}$ bei einer Schicht von $0,025 \mathrm{~mm}(=1 / \mathrm{mm})$ Tiefe be stimmt. Um die Zahl auf $1 \mathrm{cmm}$ zu berechnen, hat man die gefundene Zahl mit der Tiefe und mit der Verdünnung zu multiplizieren, d. h. für eine Verdünnung von 1:10 mit 400. Auch hier wird selbstverständlich das Resultat um so zuverlässiger, je mehr Reihen man durchzählt. Die gesamte Zahl hat man dann durch diejenige der gezählten Rechtecke zu dividieren und das Resultat wie oben mit $400 \mathrm{zu}$ multiplizieren. Es ist leicht zu verstehen, daB, wenn man z. B. 40 Reihen gezählt hat, was genau $1 \mathrm{cmm}$ ausmacht (und damit auch strengsten Anforderungen ent spricht), man eigentlich nur die gefundene Gesamtzahl mit der Vel. dünnung $(10$ z. B.) zu multiplizieren hat, um die Zahl der in $1 \mathrm{cmm}$ enthaltenen Zellen zu bekommen.

Man kann selbstverständlich auch von einem Rechteck in ein anderes überspringen und dann in dem letzteren weiterzählen, wie man ja auch, wenn man am Ende eines Rechtecks angelangt ist, auf ein anderes übergehen muB.

Die Quadrateinteilung kommt also in gewöhnlichen Fällen für die Rechnung nicht in Betracht. Sie gibt vielmehr nur Anhaltspunkte, um z. B. die gleiche Zelle nicht zweimal zu zählen, etc.

Sie kann aber auch sehr vorteilhaft werden, wenn man bei sehr starker Leukozytenvermehıung zählt. Dann gibt sie die Möglichkeit, auf stärkere Verdünnungen, die in solchen Fällen bei anderen Kammer' unumgänglich sind, zu verzicliten. Ist die Leukozytenvermehrung außerordentliclı stark, dann kann man sich der ,durchquerten“ Quadrate bedienen, so, wie es bald für die Erythrozytenzählungen zu besprechen sein wird. Eine Leukozytenvermehrung von 200000 pro $\mathrm{cmm}$ gib 5 Zellen pro nicht durchquertes Quadrat, also ungefähr 1 Zelle in jeder feinsten Einteilung der ,,durcliquerten“" Quadrate.

b) Erythrozyten- und Blutplättchenzählung. Mall kanı sich ebenso gut der nicht ,durchquerten " wie auch der ,,durchquerten“ Rechtecke bedienen. In beiden Fällen hat man in jedem Quadrat die gleiche Zahl wie im kleinsten Quadrat der Thoma-Zeißschen Kammer bei einem normalen Menschen 13-15 Zellen. Zur genaueren Zählung bedient man sich besser der, ,durchquerten" Reihen. Die feineren Einteilungen verhindern ein zweimaliges Zählen der gleichen Zelle.

Man stellt sich auch hier, wie für Leukozytenzählung, das Ende eines Rechtecks ein, und das Präparat wird, wie oben, bis zum anderen linde verschoben. Damit hat man die Erythrozytenzahl eines Raumes, dessen Basis $1 \mathrm{qmm}$ und dessen Tiefe $0,025 \mathrm{~mm}$, d. h. ${ }^{1 / 40} \mathrm{cmm}$ beträgt. Es muß dann mit der Tiefe (40) und mit der Verdünnung (gewöhnlich 100), d. h. mit 4000 multipliziert werden.

Eine solche Zälllung, die sicher sehr genau ist, wird aber dadurch überflüssig, daß man sich hier, ohne Eintrag an Genauigkeit, auf einc Anzahl beliebig gewählter Quadrate beschränken kann. Man stellt sich z. B. irgendein Quadrat, wie gesagt, vorteilhafter eines der ,durch quertell" Reihen, ein. Dann zählt man irgendein anderes, bis man die ge wïnschte Anzahl auf die ganze Oberfläche verteilter Quadrate durch. gemustert hat. Zählt man z. B. nur 30 Quadrate, absolut berechnet also weniger als ein Viertel der Thoma-Zeißschen Kammer, so erhält man doch genauere Resultate. Die gefundene Zahl wird durch die Zall] cler gezählten Quadrate dividiert und das Resultat mit 400000 (40 Tiefe; 100 - Zalll der Quadrate auf einer Reihe; 100 - Verdünnungl multiplizier't. Selbstver'ständliclı ist es ratsanl, mehı Quadrate zu zähle:1l, evelituell 100

Dem Befund von 5200000 Zellen pro cmm entsprechen 13 Zellen auf ein nicht durchquertes Quadrat, d. h. ungefälır 3 Zellen füı diє feinste Einteilung der durehquerten.

c) Lumbalpunktate. Zellreiche Flüssigkeiten werden so ge. zählt wie die Leukozyten des Blutes. Bei zellarmen gibt der einfache Ueberblick über die Kammer, die eine Schicht von $1^{1 / 2} \mathrm{cmm}$ enthält, eine genügende Schätzung.

Bei $Z$ ählungen mit I m mersion bedient man sich selbstverständlich eines feineren Deckgläschens, das jedem Apparat zugefügt ist. Es ist vorteilhaft, sich solcher Vergrößerungen zu bedienen, bei denen nur ein einziges Quadrat im ganzen Gesichtsfeld erscheinen kann. Besitzt man nicht die dazu nötigen Objektive und Okulare, dann kann man die gewünschte Vergrößerung durch Ausziehen des Tubus bekommen (s. Fing. 1, 2, 3).

d) Für die Spezialzählung der Zellen mit $a^{\prime}$ Granula, die in meiner vor kurzem in ,Zieglers Beiträgen " cl'schienenen Arbeit beschrieben 
sind, kann man sich mit großem Vorteil der dort angegebenen Türk. schen Färbung bedienen.

e) Bei Mikrobenzählung, zur Herstellung von Vakzinen, benutzt man selbstverständlich nur die Immersion. Man verdünnt die Mikrobensuspension so stark, bis man in einem eingestellten Quadrat eine Zahl von Mikroben sieht, die klein genug ist, um gezählt werden zu können. Dann verfährt man so wie bei Erythrozytenzählungen (s. o.). Man stellt sich ein ,durchquertes“ Quadrat ein, das man mit Hilfe der feineren Einteilungen leicht durchzählen kann. Man zählt eine größere Anzahl solcher Quadrate, die man auf der ganzen Fläche beliebig wählt, und dividiert die Gesamtzahl durch die der gezählten Quadrate. Das Resultat wird mit 4000 und mit der Verdünnung multipliziert. Die gefundene Zahl stellt also die in $1 \mathrm{cmm}$ der Originalsuspension vorhandenen Mikroben dar. Daraus läßt sich dann mit Leichtigkeit berechnen, wie stark die Originalsuspension verdünnt oder konzentriert werden muß, um in $1 \mathrm{ccm}$ der Vakzine die gewünschte Zahl Mikroben zu erhalten.

Als Verdünnungsflüssigkeit für die Zählung kann man sich vorteilhaft solcher Flüssigkeiten bedienen, welche, wie das Karbolfuchsin, Antiseptikum und Farbstoff enthalten. Das Karbol wirkt auch als Beizmittel für die Farbe, was nicht zu unterschätzen ist, da man gezwungen ist, nur ganz schwache Farblösungen zu gebrauchen, um die Suspension nicht undurchsichtig zu machen. Die Zählung erfolgt am besten bei künstlichem Licht. 